\title{
Recognizing and Responding to Student Affect
}

\author{
Beverly Woolf $^{1}$, Toby Dragon ${ }^{1}$, Ivon Arroyo ${ }^{1}$, David Cooper ${ }^{1}$, \\ Winslow Burleson ${ }^{2}$, and Kasia Muldner ${ }^{2}$ \\ ${ }^{1}$ Department of Computer Science, University of Massachusetts Amherst \\ 140 Governors Drive, Amherst MA 01003, USA \\ \{dragon, bev, ivon, dcooper\} @ c. . umass . edu \\ ${ }^{2}$ School of Computer Science and Informatics/Arts, Media and Engineering, \\ Arizona State University, Tempe AZ 85287, USA \\ \{winslow. burleson, Katarzyna.Muldner\} @asu. edu
}

\begin{abstract}
This paper describes the use of wireless sensors to recognize student emotion and the use of pedagogical agents to respond to students with these emotions. Minimally invasive sensor technology has reached such a maturity level that students engaged in classroom work can us sensors while using a computer-based tutor. The sensors, located on each of 25 student's chair, mouse, monitor, and wrist, provide data about posture, movement, grip tension, facially expressed mental states and arousal. This data has demonstrated that intelligent tutoring systems can provide adaptive feedback based on an individual student's affective state. We also describe the evaluation of emotional embodied animated pedagogical agents and their impact on student motivation and achievement. Empirical studies show that students using the agents increased their math value, self-concept and mastery orientation.
\end{abstract}

Keywords: intelligent tutoring systems, wireless sensors, student emotion, pedagogical agents.

\section{Introduction}

Sophisticated methods have been developed for building the interfaces of intelligent tutoring systems, but thus far they have focused almost exclusively on the cognitive side of teaching. If computers are to interact naturally with humans, they must recognize affect and express social competencies. However, the role of affect in instruction is at best in its infancy. The possibility of tutoring systems that trace students' emotions is an attractive concept; our primary research goal is to systematically examine the relationship(s) between student affective state and desired outcomes, i.e., to identify whether a dependency exists between students' reported emotions and their learning, motivation, and attitudes toward mathematics.

Various classroom studies have linked interpersonal relationships between teachers and students to increased student motivation over the long term [1,2] Thus great interest exists to embed affective support into tutoring applications. Since affect recognition is a key aspect of tailored affective support, research has focused on automated detection of affective states in a variety of learning contexts [e.g., 3-7]. Hardware sensors have the 
potential to provide information on students' physiological responses that have been linked to various affective states [e.g., 4]. Research explores various sensors' potential for affect recognition, e.g., Burleson [14] developed a learning companion that depended on a sensor framework (incorporating a mouse, posture chair, video camera, skin conductance bracelet) to recognize and respond to student affect.

Currently there is no gold standard for labeling a person's emotional state nor responding to it. Our approach is to triangulate among three different inputs: sensor data, student self-reports, and human observation of students. While we accept that there will never be definitive categorization of a human's emotional state, we plan to use this triangulation to identify clear examples of certain emotions (frustration, flow, etc.) that can be labeled using sensor information.

\section{Recognizing Student Emotion: Physiologic Sensors}

To date, much of existing work in detecting emotion has focused on inferring students' affective states with software (e.g., machine learning) [e.g., 3, 8]. However, hardware sensors have the potential to provide information on students' physiological responses that have been linked to various affective states [e.g., 6,7]. Our sensor platform includes four physiological sensors (Fig. 1) and is currently being tested in high school computer labs, demonstrating that the platform is unobtrusive enough to be used by student in a typical setting and resource-conscious enough to run on average computer labs available to students. These sensors collect raw data about physical activity and state of a student and the challenge remains to map this data into models of emotional states and use this information productively. The sensors are similar to Burleson's more costly sensors used in previous studies [9]. We describe how our sensors compare to earlier sensors as well as some of the past uses of the earlier sensors. Major improvements focus on the overall production cost and the noninvasive nature of the sensors.

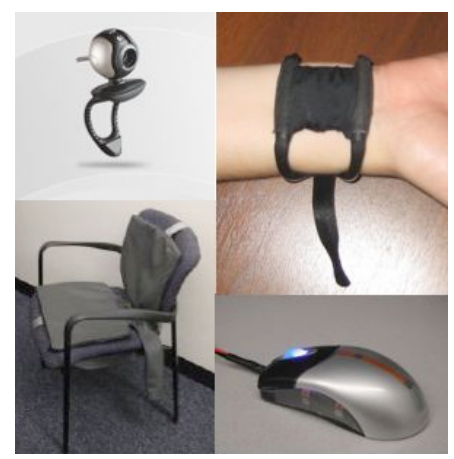

Fig. 1. Sensors used in the classroom (clockwise): mental state camera, skin conductance bracelet, pressure sensitive mouse, pressure sensitive chair

Skin Conductance Bracelet. The Affective Computing Group at the MIT Media Lab has been advancing the development of wireless wearable skin conductance sensors 
for over a decade. Various implementations include the galvactivator, a glove that could illuminate an LED when its user had heightened levels of skin conductance [10]; HandWave which used a custom built Printed Circuit Board (PCB) and 9V battery to provide blue tooth wireless transmission of skin conductance data at rates up to5 $\mathrm{Hz}[11]$.

The current system used in our research employs the next generation of Hand Wave electronics developed at MIT, providing greater reliability, lower power requirements through wireless RFID transmission, and a smaller form. This smaller form was redesigned to minimize the visual impact and increase the wearable aspects of previous versions. ASU integrated and tested these electronic components into a wearable package suitable for students in classrooms. Our version reports at $1 \mathrm{~Hz}$.

Pressure Sensitive Mouse. The pressure mouse was developed by the Affective Computing Group at MIT. It uses six pressure sensors embedded in the surface of the mouse to detect the tension in users' grip and has been used to infer elements of users' frustration [12, 13] Our endeavors replicated MIT's pressure mouse through a production of 30 units. The new design of the mouse minimized the changes made to the physical appearances of the original mouse in order to maintain a visually noninvasive sensor state.

Pressure Sensitive Chair. The posture state chair that was used in Burleson's affective Learning Companion [14, 15] utilized a donated Tek-Scan pressure system; the commercial cost of this system was and continues to be upwards of $\$ 10,000$. A software system was developed and trained to detect nine states of posture and activity level [16] and was subsequently used in conjunction with the blue-eyes camera hardware can additional software to detect levels of interest, boredom, and break-taking while students engaged in an educational game.

A greatly simplified chair sensor system was developed at ASU using a series of eight force sensitive resistors as pressure sensors dispersed throughout the seat and back of a readily available seat cover cushion. This posture chair sensor was developed at ASU at an approximate cost of $\$ 500$ per chair for a production volume of 30 chairs.

Mental State Camera. The facial expression recognition system used in Burleson's Affective Learning Companion [14,15] utilized IBM Research's Blue-Eyes camera hardware. Which used a digital camera board with adjustable focused lens augmented with a custom PCB that provided differential LED illumination (e.g. conical illumination in close circumferential positions around the lens, used to obtain retroreflection from users' retina; and axial illumination that reflects of the front surface of users' eyeballs). Using the differential images to detect pupil location, pattern recognition models were trained to detect head nod and shake behaviors, blinking, pupil dilation, mouth fidgets and smiles. One limitation of this system was the need for the integration of the custom PCB with the camera, adding to the expense; a second limitation was its development in Linux, which made it less compatible with most operating systems found in schools today.

In our current research we use a standard web-camera to obtain 30fps at 320x240 pixels. This is coupled with El Kaliouby's MindReader applications [17]. We developed a Java Native Interface (JNI) wrapper around the MindReader library. The interface starts a version of the MindReader software, and can be queried at any time 
to get the most recent mental state values that have been computed by the library. In the version used in the experiments, only the six mental state features were available, but in future versions we can train it on new mental states.

In our framework, each feature source from each student is a separate stream of data. Hence we have streams of data which each report asynchronously and at different rates. In order to merge all of the data sources, an ID from each student, and a time of the report was needed from each source. We have a database table with a row for every time stamp and wrist ID pair, and a column for each reported sensor value and tutor data value. Each cell in a row represents the latest report of the data source.

\section{Testbed Application: Wayang}

The sensor data collection and analysis system described above is stand-alone software and can provide input for any system. Currently we are using it in tandem with Wayang Outpost, a multimedia tutoring system for high school geometry and algebra, see Fig. 2. [18, 19] Students learn by working through problems both in a real-world context and in a test-like environment in preparation for standardized testing such as the SAT and other state exams. Prior research has shown that examining a student's affect is critical to learning mathematics, since affect (particularly frustration and boredom) has been found to be an important factor in student learning [2] is adaptive in that it iterates through different topics (e.g. pythagorean theorem) and multiple hints based on student need. Within each topic section, Wayang adjusts the difficulty of problems provided depending on past student performance. Students are presented with a problem and asked to choose the solution from a list of multiple-choice options. As students solve problems, they may ask the tutor for one or several multimedia hints via the help button, which combine text messages, audio and animations. Wayang has been used with thousands of students in the past and has demonstrated improved learning gains in state standard exams [20]. Wayang collects student interaction features in order to predict the level of effort of each student.

\section{Experiments}

We conducted three studies during Fall 2008 involving the use of sensors and Wayang Outpost. One study involved 35 students in a public high school (HS) in Massachusetts; another involved 29 students in the University of Massachusetts (UMASS); and the final study involved 29 undergraduates from Arizona State University (AZ). In the HS and UMASS studies, students used the software as part of their regular math class for 4-5 days and covered topics in the traditional curriculum. In the AZ lab study, students came into a lab for a single session.

The three experiments yielded the results of 588 Emotional Queries from 80 students who were asked about their emotion, e.g., "How confident do you feel?" The response was a scale 1-5 and the queries separated into four emotion variables: 149 were about confidence/anxiety, 163 about excitement/depression, 135 about 
interest/boredom, and 141 about frustrated/not frustrated. 16 of the student responses gave no answer to the Emotional Query. Models were created to automatically infer student emotions from physiological data from the sensors. Students produced selfreports of emotions and all queries include valid data from at least one sensor.

In order to select a subset of the available features, a stepwise regression was done with each of the emotions as the dependent variable, and tutor and sensor features as the independent variables. Since some students had missing sensor data, separate models were run pairing the tutor with one sensor at a time, and then finally with all of the sensors. Results from the regression show that the best models for confident, frustrated, and excited came from the subset of examples where all of the sensor data was available, and the best model for interested came from the subset of examples with mouse data available. Summaries of student physiological activity, in particular data streams from facial detection software, helped to predict more than $60 \%$ of the variance of students emotional states, which is much better than predicting emotions from other contextual variables from the tutor, when these sensors are absent. In order for the user model system to provide feedback to the tutor, the available sensor and tutor features can be put into a classifier and report when a user is likely to report a high value of a particular emotion, Table 1. This likelihood could reduce and possibly eliminate the need for querying the user of their affective state. To test the efficacy of this idea, we made a classifier based on each linear model in the table. For each model we performed leave-one-student-out cross validation. We recorded the number of True Positives, False Negatives, True Negatives, and False Positives at each test. Table 1 shows results from the best classifier of each emotion in terms of Accuracy. The best classification results are obtained by only training on examples that are not in the middle (scale of 1-5). This is likely the case because the middle values (3) show student indifference.

Table 1. This shows results of the best classifier of each emotional response. Accuracy of no classifier is a prediction that the emotional state is always not high. Values in parentheses include the middle values in the testing set as negative examples.

\begin{tabular}{c|cc|cc|cc}
\hline Classifier & \multicolumn{2}{|c|}{ True } & False & True & False & Accuracy (\%) \\
& Pos. & Pos. & Neg. & Neg. & & No Classifier \\
\hline Confident All & $28(28)$ & $5(24)$ & $10(16)$ & $1(1)$ & $86.36(63.77)$ & $34.09(57.97)$ \\
Frustrated All & $3(3)$ & $0(0)$ & $46(58)$ & $7(7)$ & $87.5(89.7)$ & $82.14(85.29)$ \\
Excited Wrist & $25(25)$ & $9(37)$ & $25(40)$ & $5(5)$ & $78.1(60.7)$ & $53.12(71.96)$ \\
Interested Mouse & $24(25)$ & $4(19)$ & $28(53)$ & $7(7)$ & $82.54(74.76)$ & $50.79(69.90)$ \\
\hline
\end{tabular}

\section{Responding to Student Affect}

Providing empathy or support strongly correlates with learning [21, 22] and the presence of someone who cares, or at least appears to care, can be motivating. Various studies have linked interpersonal relationships between teachers and students to motivational outcomes $[23 ; 1]$. Can this noted human relationship be reproduced, 
in part, by apparent empathy from a computer character? Apparently the answer is yes [24]. People seem to relate to computers in the same way they relate to humans and some relationships are identical to real social relationships [25]. For example, students continue to engage in frustrating tasks on a computer significantly longer after an empathetic computational response [26], have immediately lowered stress level (via skin conductance) after empathy and after apology [27], and relational skills improve long-term ratings of caring, trust, respect, desire to keep working [28]. Computer agents impact student learning, affect and motivation based on gender, ethnicity and realism of the agent [29].

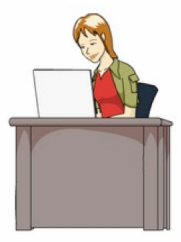

Confident

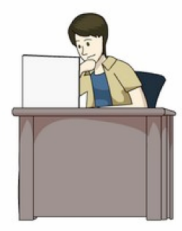

Focused

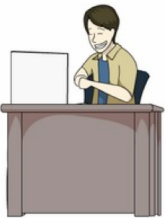

Excited

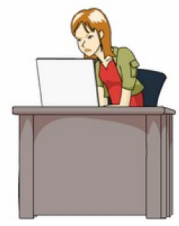

Frustrated

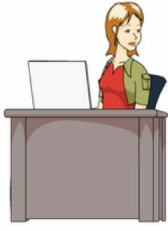

Bored

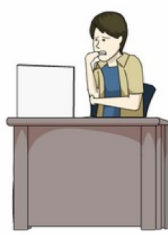

Anxious

Fig. 2. Pedagogical Agents act out their emotion and talk with the student expressing full sentences of cognitive, meta-cognitive and emotional feedback

This is not to say that the inferences, movements and interventions of computer agents can exactly replace those of people, nor can peer theories exactly map to the human peer-tutoring case; however, though computer control does allow for careful testing of hypotheses about how to use virtual peer support for learning [23].

Wayang includes gendered learning companions, see Fig. 2, that provide support and encouragement, emphasize the importance of perseverance, express emotions and offer strategies (e.g., "Use the help function"). These learning companions (LCs) are empathetic in that they visually reflect the last emotion reported by the student (queried within the system every five minutes); they act out their emotion and talk with students expressing full sentences of meta-cognitive and emotional feedback. They are non-intrusive -- they work on their own computer to solve the problem at hand, and react only after the student has answered the question. Agents respond with some of Carole Dweck's [30] recommendations about disregarding success and valuing effort. This adds a new dimension to the traditional feedback regarding success/no-success generally given to students. 


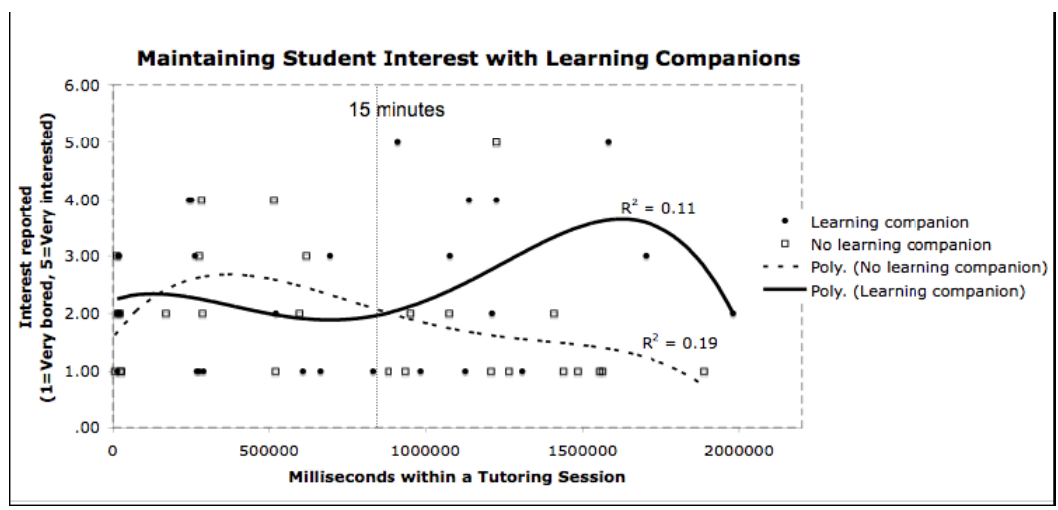

Fig. 3. Learning companions make mathematics more interesting. Students with LCs reported more interest in math sessions. Lines represent best-fit curves, which in this case are 4th degree polynomials.

We measured the impact of LCs on student motivation and achievement (Woolf et al., submitted) and integrated controlled exploration of their communicative factors (facial expression and mirroring postures) as the student/agent relationship developed. Empirical studies show that students who use LCs increased their math value (e.g., questions such as "Mathematics is an important topic"), self-concept (e.g., "I am good in mathematics") and mastery orientation (Woolf et al., submitted). Students tend to become more bored (less interested) towards the end of any instructional session. Yet the student using LCs maintain higher levels of interest and reduced boredom after 15 minutes of tutor use, see Fig. 3. They reported a higher mean confidence, interest and excitement. Despite the fact these results were not significant, this relative advantage for LCs indicates that they might alleviate students' boredom as the session progresses.

\section{Discussion and Future Work}

This paper described the use of wireless sensors to recognize student emotion and the use of pedagogical agents to respond to students. We presented a user model framework to predict emotional self-concept. The framework works in classrooms of up to 25 students with four sensors per student. By using stepwise regression we have isolated key features for predicting user emotional responses to four categories of emotion. This is backed up by cross validation, and shows a small improvement using a very basic classier. This data has demonstrated that intelligent tutoring systems can provide adaptive feedback based on an individual student's affective state. We also described the evaluation of emotional embodied animated agents and their impact on student motivation and achievement.

There are a number of places for improvement in our system. The first is that we used summary information of all sensor values. We may find better results by considering the time series of each of these sensors. In addition, the software in the Mental State Camera can be trained for new mental states. This is one avenue of 
future work. Another place for improvement is to look at individual differences in the sensors. Creating a baseline for emotional detection before using the tutor system could help us to better interpret the sensor features.

Emotional predictions from sensors and agents as described above are a first step towards personalized feedback for students in classroom environments. We propose that the tutor will identify desirable (e.g. flow) and non-desirable (e.g. boredom) student states. Different interventions will be tested in an attempt to keep students in desirable states as much as possible (e.g. a confused student might be invited to slow down, reread the problem and ask for a hint). Part of this approach includes embedding a user model into the tutor to provide instructional recommendations. Interventions algorithms are being developed based on tutor predictions, e.g. mirror student emotion, support student effort, provide more immediate feedback on student progress, and allow students increased control of their experience.

Acknowledgement. This research was funded by awards from the National Science Foundation, 0705554, IIS/HCC Affective Learning Companions: Modeling and supporting emotion during teaching, Woolf and Burleson (PIs) with Arroyo, Barto, and Fisher and the U.S. Department of Education to Woolf, B. P. (PI) with Arroyo, Maloy and the Center for Applied Special Technology (CAST), Teaching Every Student: Using Intelligent Tutoring and Universal Design To Customize The Mathematics Curriculum. Any opinions, findings, conclusions or recommendations expressed in this material are those of the authors and do not necessarily reflect the views of the funding agencies.

We acknowledge contributions to the system development from Rana el Kaliouby, Ashish Kapoor, Selene Mota and Carson Reynolds. We also thank Joshua Richman, Roopesh Konda, and Assegid Kidane at ASU for their work on sensor manufacturing. We thank Jerry Chen and William Ryan for their contributions to agent development.

\section{References}

1. Wentzel, K., Asher, S.R.: Academic lives of neglected, rejected, popular, and controversial children. Child Development 66, 754-763 (1995)

2. Royer, J.M., Walles, R.: Influences of gender, motivation and socioeconomic status on mathematics performance. In: Berch, D.B., Mazzocco, M.M.M. (eds.) Why is math so hard for some children, pp. 349-368. Paul. H. Brookes Publishing Co., Baltimore (2007)

3. Conati, C., Maclare, H.: Evaluating a Probabilistic Model of Student Affect. In: Lester, J.C., Vicari, R.M., Paraguaçu, F. (eds.) ITS 2004. LNCS, vol. 3220, pp. 55-66. Springer, Heidelberg (2004)

4. D'Mello, S., Graesser, A.: Mind and Body: Dialogue and Posture for Affect Detection in Learning Environments. Paper presented at the Frontiers in Artificial Intelligence and Applications (2007)

5. McQuiggan, S., Lester, J.: Diagnosing Self-Efficacy in Intelligent Tutoring Systems: An Empirical Study. In: Ikeda, M., Ashley, K., Chan, T.W. (eds.) Eighth International Conference on Intelligent Tutoring Systems, Jhongli, Taiwan (2006)

6. Graesser, A.C., Chipman, P., King, B., McDaniel, B., D’Mello, S.: Emotions and Learning with AutoTutor. In: Luckin, R., Koedinger, K., Greer, J. (eds.) 13th International Conference on Artificial Intelligence in Education (AIED 2007), pp. 569-571. IOS Press, Amsterdam (2007) 
7. D’Mello, S.K., Picard, R.W., Graesser, A.C.: Towards an Affect-Sensitive AutoTutor. Special issue on Intelligent Educational Systems. IEEE Intelligent Systems 22(4), 53-61 (2007)

8. Conati, C., Mclaren, H.: Evaluating A Probabilistic Model of Student Affect. In: Lester, J.C., Vicari, R.M., Paraguaçu, F. (eds.) ITS 2004. LNCS, vol. 3220, pp. 55-66. Springer, Heidelberg (2004)

9. Burleson, W.: Affective Learning Companions: Strategies for Empathetic Agents with Real-Time Multimodal Affective Sensing to Foster Meta-Cognitive Approaches to Learning, Motivation, and Perseverance. MIT PhD thesis (2006), http://affect.media.mit.edu/pdfs/06.burleson-phd.pdf

10. Picard, R.W., Scheirer, J.: The galvactivator: A glove that senses and communicates skin conductivity. In: 9th International Conference on Human-Computer Interaction, New Orleans, August 2001, pp. 1538-1542 (2001)

11. Strauss, M., Reynolds, C., Hughes, S., Park, K., McDarby, G., Picard, R.: The handwave bluetooth skin conductance sensor. In: Tao, J., Tan, T., Picard, R.W. (eds.) ACII 2005. LNCS, vol. 3784, pp. 699-706. Springer, Heidelberg (2005)

12. Qi, Y., Picard, R.: Context-sensitive bayesian classifiers and application to mouse pressure pattern classification. In: 16th International Conference on Pattern Recognition, Proceedings, vol. 3, pp. 448-451 (2002)

13. Dennerlein, J., Becker, T., Johnson, P., Reynolds, C., Picard, R.W.: Frustrating computer users increases exposure to physical factors. In: Proceedings of International Ergonomics Association, Seoul, Korea, pp. 24-29 (2003)

14. Mota, S., Picard, R.W.: Automated posture analysis for detecting learner's interest level. In: Computer Vision and Pattern Recognition Workshop, vol. 5, p. 49 (2003)

15. Kapoor, A., Burleson, W., Picard, R.W.: Automatic prediction of frustration. International Journal of Human-Computer Studies 65(8), 724-736 (2007)

16. Burleson, W., Picard, R.W.: Gender-specific approaches to developing emotionally intelligent learning companions. IEEE Intelligent Systems 22(4), 62-69 (2007)

17. el Kaliouby, R.: Mind-reading Machines: the automated inference of complex mental states from video. PhD thesis, University of Cambridge (2005)

18. Arroyo, I., Cooper, D., Burleson, W., Woolf, B.P., Muldner, K.: Empathetic Pedagogical Agents. Submitted to AIED (2009)

19. Arroyo, I., Ferguson, K., Johns, J., Dragon, T., Mehranian, H., Fisher, D., Barto, A., Mahadevan, S., Woolf, B.: Repairing Disengagement With Non Invasive Interventions. In: International Conference on Artificial Intelligence in Education, Marina del Rey, CA (2007)

20. Dragon, T., Arroyo, I., Woolf, B.P., Burleson, W., El Kaliouby, R., Eydgahi, H.: Viewing Student Affect and Learning through Classroom Observation and Physical Sensors. In: Woolf, B.P., Aïmeur, E., Nkambou, R., Lajoie, S. (eds.) ITS 2008. LNCS, vol. 5091, pp. 29-39. Springer, Heidelberg (2008)

21. Graham, S., Weiner, B.: Theories and principles of motivation. In: Berliner, D., Calfee, R. (eds.) Handbook of Educational Psychology, pp. 63-84. Macmillan, New York (1996)

22. Zimmerman, B.J.: Self-Efficacy: An Essential Motive to Learn. Contemporary Educational Psychology 25, 82-91 (2000)

23. Picard, R.W., Papert, S., Bender, W., Blumberg, B., Breazeal, C., Cavallo, D., Machover, T., Resnick, M., Roy, D., Strohecker, C.: Affective Learning-A Manifesto. BT Journal 2(4), 253-269 (2004)

24. Bickmore, T., Picard, R.W.: Establishing and Maintaining Long-Term Human-Computer Relationships. Transactions on Computer-Human Interaction 12(2), 293-327 (2004) 
25. Reeves, B., Nass, C.: The media equation: How people treat computers, television and new media like real people and places. CSLI, New York (1998)

26. Klein, J., Moon, Y., Picard, R.W.: This Computer Responds to User Frustration: Theory, Design, Results, and Implications. Interacting with Computers 14(2), 119-140 (2002)

27. Prendinger, H., Ishizuka, M.: The Empathic Companion: A Character-Based Interface that Addresses Users' Affective States. Applied Artificial Intelligence 19(3-4), 267-285 (2005)

28. Bickmore, T., Picard, R.W.: Establishing and Maintaining Long-Term Human-Computer Relationships. Transactions on Computer-Human Interaction 12(2), 293-327 (2004)

29. Baylor, A.: The Impact of Pedagogical Agent Image on Affective Outcomes. In: Proceedings of Workshop on Affective Interactions: Computers in the Affective Loop, International Conference on Intelligent User Interfaces, San Diego, CA (2005)

30. Dweck, C.: Messages that motivate: How praise molds students' beliefs, motivation, and performance (In Surprising Ways). In: Aronson, J. (ed.) Improving academic achievement. Academic Press, New York (2002) 